\title{
STAR FORMATION ON SUBKILOPARSEC SCALE TRIGGERED BY NON-LINEAR PROCESSES IN NEARBY SPIRAL GALAXIES
}

\author{
Rieko Momose $^{1,2,10}$, Jin Koda ${ }^{3}$, Robert C. Kennicutt JR ${ }^{4}$, Fumi Egusa ${ }^{5}$, Daniela Calzetti ${ }^{6}$, Guilin Liu ${ }^{6,11}$, \\ Jennifer Donovan Meyer ${ }^{3,12}$, Sachiko K. Okumura ${ }^{2,13}$, Nick Z. Scoville ${ }^{7}$, Tsuyoshi Sawada ${ }^{2,8}$, and Nario Kuno ${ }^{9}$ \\ ${ }^{1}$ Department of Astronomy, University of Tokyo, Hongo, Bunkyo-ku, Tokyo 113-0033, Japan; momo.s.rieko@ @ao.ac.jp \\ ${ }^{2}$ National Astronomical Observatory of Japan, Mitaka, Tokyo 181-8588, Japan \\ ${ }^{3}$ Department of Physics and Astronomy, Stony Brook University, Stony Brook, NY 11794-3800, USA \\ ${ }^{4}$ Institute of Astronomy, University of Cambridge, Cambridge CB3 OHA, UK \\ ${ }^{5}$ Institute of Space and Astronautical Science, Japan Aerospace Exploration Agency, Chuo-ku, Sagamihara, Kanagawa 252-5210, Japan \\ ${ }^{6}$ Astronomy Department, University of Massachusetts, Amherst, MA 01003-9305, USA \\ ${ }^{7}$ Astronomy Department, California Institute of Technology, MC 249-17, 1200 East California Boulevard, Pasadena, CA 91125, USA \\ ${ }^{8}$ Joint ALMA Observatory, Alonso de Córdova 3107, Vitacura, Santiago 763-0355, Chile \\ ${ }^{9}$ Nobeyama Radio Observatory, National Astronomical Observatory of Japan, Nobeyama, Minamimaki, Minamisaku, Nagano 384-1305, Japan \\ Received 2013 March 28; accepted 2013 June 23; published 2013 July 10
}

\begin{abstract}
We report a super-linear correlation for the star formation law based on new $\mathrm{CO}(J=1-0)$ data from the CARMA and NOBEYAMA Nearby-galaxies (CANON) CO survey. The sample includes 10 nearby spiral galaxies, in which structures at sub-kpc scales are spatially resolved. Combined with the star formation rate surface density traced by $\mathrm{H} \alpha$ and $24 \mu \mathrm{m}$ images, $\mathrm{CO}(J=1-0)$ data provide a super-linear slope of $N=1.3$. The slope becomes even steeper $(N=1.8)$ when the diffuse stellar and dust background emission is subtracted from the H $\alpha$ and $24 \mu \mathrm{m}$ images. In contrast to the recent results with $\mathrm{CO}(J=2-1)$ that found a constant star formation efficiency (SFE) in many spiral galaxies, these results suggest that the SFE is not independent of environment, but increases with molecular gas surface density. We suggest that the excitation of $\operatorname{CO}(J=2-1)$ is likely enhanced in the regions with higher star formation and does not linearly trace the molecular gas mass. In addition, the diffuse emission contaminates the SFE measurement most in regions where the star formation rate is law. These two effects can flatten the power-law correlation and produce the apparent linear slope. The super-linear slope from the $\operatorname{CO}(J=1-0)$ analysis indicates that star formation is enhanced by non-linear processes in regions of high gas density, e.g., gravitational collapse and cloud-cloud collisions.
\end{abstract}

Key words: galaxies: ISM - galaxies: spiral - galaxies: star formation - ISM: molecules - radio lines: galaxies

Online-only material: color figures

\section{INTRODUCTION}

The relation between star formation rate (SFR) surface density $\left(\Sigma_{\mathrm{SFR}}\right)$ and gas surface density $\left(\Sigma_{\mathrm{gas}}\right)$ is important for our understanding of the star formation mechanism in galaxies. Schmidt (1959) suggested a power-law correlation between $\Sigma_{\mathrm{SFR}}$ and $\Sigma_{\text {gas }}, \Sigma_{\mathrm{SFR}} \propto \Sigma_{\text {gas }}^{N}$. Kennicutt (1998a, 1998b, hereafter K98a, K98b) expanded the early study substantially and found a super-linear correlation with $N=1.4$ by combining atomic (HI) and molecular (CO $J=1-0)$ data, and calculating SFR using $\mathrm{H} \alpha$ and infrared data. This correlation is often called the Kennicutt-Schmidt law (hereafter, the K-S law). Extensive reviews can be found in K98b and Kennicutt \& Evans (2012).

The index of the K-S law $(N)$ has been a primary indicator of the mechanism of star formation. Recent studies of the $\mathrm{K}-\mathrm{S}$ law investigated the index at sub-kpc scales, approaching the intrinsic scale of star formation, i.e., the sizes of giant molecular clouds (GMCs) or giant molecular associations.

\footnotetext{
${ }^{10}$ Current address: Institute for Cosmic Ray Research, University of Tokyo, 5-1-5 Kashiwano-ha, Kashiwa city, Chiba 277-8582, Japan; momo@icrr.u-tokyo.ac.jp

${ }^{11}$ Current address: Center for Astrophysical Sciences, Johns Hopkins University, 3400 North Charles Street, Baltimore, MD 21218-2686, USA

${ }^{12}$ Current address: National Radio Astronomy Observatory, 520 Edgemont Rd., Charlottesville, VA, USA

${ }_{13}$ Current address: Department of Mathematical and Physical Sciences, Japan Women's University, 2-8-1 Mejirodai, Bunkyo-ku, Tokyo 112-8681, Japan
}

Bigiel et al. (2008, hereafter B08) derived $N \sim 1$ from the correlation between $\Sigma_{\mathrm{SFR}}$ and the molecular gas surface density $\Sigma_{\mathrm{H}_{2}}$ estimated from $\mathrm{CO}(J=2-1)$ data for seven nearby spiral galaxies. They suggested that a linear correlation is evident in regions of high gas surface densities where the gas is typically molecular $\left(\geqslant 10 M_{\odot} \mathrm{pc}^{-2}\right)$. Several studies using $\mathrm{CO}(J=2-1)$ also showed a linear correlation (e.g., Leroy et al. 2008; Schruba et al. 2011), including one which combined single-dish $\mathrm{CO}(J=$ $2-1)$ data with interferometric $\mathrm{CO}(J=1-0)$ data (Rahman et al. 2011). These $\operatorname{CO}(J=2-1)$ studies analyzed a substantial number of nearby galaxies, though it should be recognized that some studies based on $\mathrm{CO}(J=1-0)$ data showed a super-linear (power-law) correlation, rather than a linear correlation (e.g., Wong \& Blitz 2002; Kennicutt et al. 2007; Liu et al. 2011).

$\mathrm{CO}(J=1-0)$ is a better calibrated tracer of the bulk molecular gas in spiral galaxies. While $\mathrm{CO}(2-1)$ emission is sensitive to density and temperature changes (Koda et al. 2012), the $\mathrm{CO}(1-0)$-to- $\mathrm{H}_{2}$ conversion factor is more stable since their changes compensate each other to some extent (Scoville \& Sanders 1987) as empirically demonstrated (Bolatto et al. 2008, 2013; Donovan Meyer et al. 2012). However, there is no systematic study of the K-S law on sub-kpc scale using $\mathrm{CO}(J=$ $1-0)$ for a large number of galaxies. A recent study by Rahman et al. (2012) used $\mathrm{CO}(J=1-0)$ data from an interferometer (but no single-dish data). The flux measured by an interferometer is only reliable when an object of interest is very compact and 
isolated. In any extended structures and their surrounding areas (e.g., spiral arms, inter-arms), the measured fluxes are uncertain. Without access to single-dish data, we don't know how much flux is resolved out in the interferometry. Such a systematic bias hinders a study of the K-S law with interferometer-only data, as star-forming regions are located predominately along spiral arms (e.g., Scoville et al. 2001).

We study the K-S law on sub-kpc scales using new $\mathrm{CO}(J=$ 1-0) data obtained by the Nobeyama $45 \mathrm{~m}$ single-dish telescope (NRO45) and CARMA. In this Letter, we discuss only the correlation of $\Sigma_{\mathrm{SFR}}$ with $\Sigma_{\mathrm{H}_{2}}$, but not with the total gas surface density $\Sigma_{\text {gas }}$. The gas phase is predominantly molecular in regions where $\Sigma_{\text {gas }}$ is above $10 M_{\odot} \mathrm{pc}^{-2}$ (B08), and the correlation holds even when $\Sigma_{\mathrm{H}_{2}}$ alone is analyzed (e.g., Kennicutt et al. 2007; B08).

In this Letter, we find a non-linear index of the K-S law in our spatially resolved analyses (on sub-kpc scales) and discuss the star formation mechanisms. We describe the data and methods of this study in Section 2 and present results in Section 3. In Section 4, we compare our new results with the previous studies and discuss star formation mechanisms.

\section{DATA AND METHOD OF THE STUDY}

\subsection{Molecular Gas Surface Density}

We use $\operatorname{CO}(J=1-0)$ data from the CARMA and NOBEYAMA Nearby-galaxies (CANON) $\mathrm{CO}(J=1-0)$ survey (J. Koda et al. in preparation; see also Donovan Meyer et al. 2013). This survey combines high-resolution interferometer data (CARMA) and total power single-dish data (NRO45), providing high-fidelity, high-resolution $\mathrm{CO}(J=1-0)$ data for nearby spiral galaxies. The single-dish data are critical to the flux measurements. For instance, the flux in a CARMA-alone map varies systematically with galactic structures; in the case of M 51 (Koda et al. 2011), the recovered flux is as low as $\sim 20 \%$ in inter-arm regions and as high as $\sim 100 \%$ in spiral arms.

We analyze 10 galaxies from the CANON sample (NGC 3521, 3627, 4254, 4303, 4321, 4736, 4826, 5055, 5194, and 6946). We convert the $\mathrm{CO}(J=1-0)$ integrated intensity to $\Sigma_{\mathrm{H}_{2}}$ using the standard $X_{\mathrm{CO}}=2.0 \times 10^{20} \mathrm{~cm}^{-2}\left(\mathrm{~K} \mathrm{~km} \mathrm{~s}^{-1}\right)^{-1}$ (Strong \& Mattox 1996; Dame et al. 2001), since all our samples are late-type spiral galaxies whose metallicities are nearly the value of solar metallicity (Moustakas et al. 2010).

\subsection{Star Formation Rate}

We combine $\mathrm{H} \alpha$ and $24 \mu \mathrm{m}$ images to estimate $\Sigma_{\mathrm{SFR}}$ using Equation (7) in Calzetti et al. (2007). Most $\mathrm{H} \alpha$ and all $24 \mu \mathrm{m}$ images are obtained from the Spitzer Infrared Nearby Galaxies Survey (SINGS) archive (Kennicutt et al. 2003). We also use some $\mathrm{H} \alpha$ images (NGC 4303, 4736, and 4826) obtained via the NASA Extragalactic Database (Knapen et al. 2004; Dale et al. 2009).

We discuss the effect of local background (BG) emission on the SFR estimate and investigate the K-S law with and without BG subtraction. The origin of $\mathrm{H} \alpha \mathrm{BG}$ may be leaked photons from distant $\mathrm{H}$ II regions (a.k.a. diffuse ionized gas; e.g., Ferguson et al. 1996). The BG of $24 \mu \mathrm{m}$ emission may be the radiation from small dust grains heated by old stars (e.g., Draine \& Li 2007). Such BG emission is not related to recent star formation events, and therefore, if present, should be removed in the SFR estimate. The BG subtraction affects the index of the K-S law significantly. The BG subtraction is not quite straightforward, and various techniques have been suggested (e.g., Rahman et al. 2011; Liu et al. 2011). In this study, we adopt the technique introduced by Liu et al. (2011), in which they divided $\mathrm{H}$ II regions and the diffuse $\mathrm{BG}$ emission using the publicly available IDL routine H I I phot (Thilker et al. 2000), and used the output image as the BG image which is generated by the interpolation from the $\mathrm{BG}$ pixels around $\mathrm{H}$ II regions and surface-fitting to estimate BG emission embedded in the $\mathrm{HII}$ regions. We also carry out smoothing of the $\mathrm{BG}$ image in the same manner as Liu et al. (2011).

We find the BG fractions of $\mathrm{H} \alpha$ and $24 \mu \mathrm{m}$ to be $33 \%-57 \%$ and $39 \%-66 \%$ of the total luminosities, respectively. These are consistent with previous results $(\sim 30 \%-50 \%$ Lyman photon leakage from H II regions (Ferguson et al. 1996), and the $24 \mathrm{~m}$ BG of 30\%-40\% in galactic centers and $20 \%$ in disks (Verley et al. 2009)). Draine et al. (2007) also found $88 \%$ of infrared emission coming from regions of very low stellar radiation fields, presumably not associated with local star formation, while Leroy et al. (2012) found a smaller fraction $(\sim 20 \%)$. There may be some room for debate on the actual BG fractions (e.g., dependences on local radiation field, the amount of underlying dust, dust grain compositions, etc.). However, their effects on the index $N$ is simple and systematic. This will be discussed in Section 3. In general, the larger the BG subtraction, the steeper the index. The index derived without BG subtraction provides the lower limit.

\subsection{Fitting the Correlation}

We apply a pixel-to-pixel analysis and derive the power index of the K-S law. We use data above $3 \sigma$ significance in both $\Sigma_{\mathrm{H}_{2}}$ and $\Sigma_{\mathrm{SFR}}$. We adopt two sampling sizes, i.e., 750 and $500 \mathrm{pc}$; the former is the scale used in the previous $\operatorname{CO}(J=2-1)$ study (B08) and the latter is a resolution similar to the one in Kennicutt et al. (2007). These pixel sizes are much larger than the pointspread function or beam size of $24 \mu \mathrm{m}\left(\sim 6^{\prime \prime}\right)$ and CO (1".9 $\sim$ 4..8) images.

We fit the data in a logarithmic space:

$$
\log \left(\Sigma_{\mathrm{SFR}}\right)=A_{\text {fit }}+N \times \log \left(\Sigma_{\mathrm{H}_{2}}\right)
$$

where $A_{\text {fit }}$ is an intercept and $N$ is the index of the K-S law. We use the FITEXY routine (Press et al. 1992), which accounts for measurement errors along both $x$ - and $y$-axes, thus providing more robust regression results, and has been used in previous studies (Kennicutt et al. 2007; Rahman et al. 2011). When comparing with B08, we also use the OLS bisector method (Isobe et al. 1990) adopted by B08, which returns a bisector line in $x$ - and $y$-axes without the errors taken into account. We note that the choice of $\mathrm{CO}$ threshold surface density (i.e., $3 \sigma$ ) affects little; we repeated the analysis below with various thresholds and obtained the same conclusions.

\section{RESULTS}

We smooth the $\operatorname{CO}(J=1-0)$ data to a $750 \mathrm{pc}$ resolution (i.e., matching the resolution for all galaxies at different distances) and fit the K-S relation. This is the resolution used in the $\mathrm{CO}(J=$ 2-1) study by B08, and our results can be directly compared with the linear correlation $N \sim 1$ of B08 (more precisely, $N=1.0 \pm$ 0.1 and $A_{\text {fit }}=-2.1 \pm 0.2$ ). We combine all the data points from all galaxies to fit a line using measurement errors as weights. B08 did not subtract the extended BG emission, and therefore, in our first analysis, we do not make the subtraction from either the $\mathrm{H} \alpha$ or $24 \mu \mathrm{m}$ images. A fit with either FITEXY or the $O L S$ bisector provides a super-linear slope of $N=1.3 \pm 0.06$ and $A_{\mathrm{fit}}$ $=-3.6 \pm 0.06$ (Figure 1(a)), steeper than the linear slope of B08 
(a)

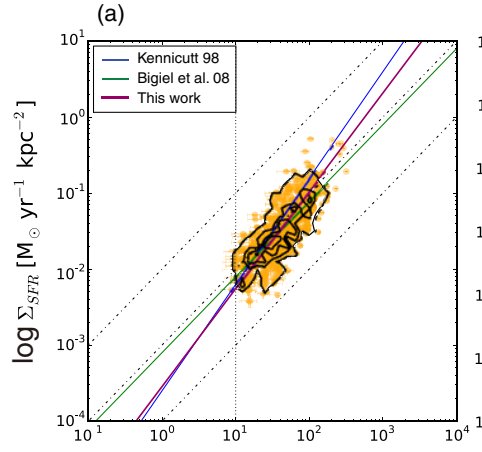

(b)

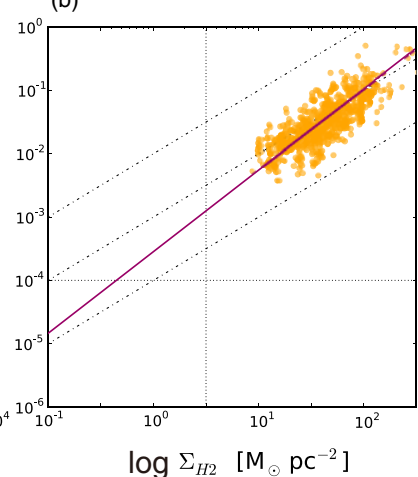

(c)

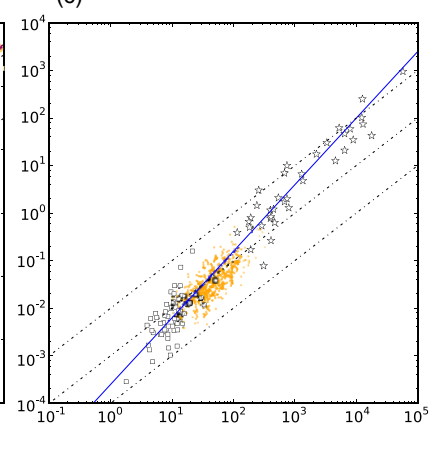

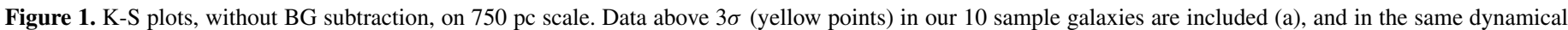

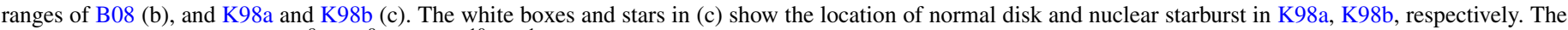

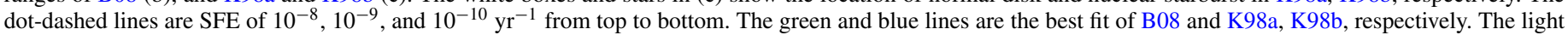
purple straight line is best fit through our data. The overlaid black contours are density distributions of the data points.

(A color version of this figure is available in the online journal.)

Table 1

Fitted Parameters of Best-fit Linear Regressions

\begin{tabular}{lcc}
\hline \hline Scale & $A_{\text {fit }}$ & $N$ \\
\hline 750 pc & $-3.6 \pm 0.06$ & $1.3 \pm 0.06$ \\
500 pc (1) & $-3.6 \pm 0.09$ & $1.3 \pm 0.06$ \\
500 pc (2) & $-5.0 \pm 0.17$ & $1.8 \pm 0.10$
\end{tabular}

Note. 500 pc scale (1) without and (2) with BG subtractions.

(see Table 1). Our power-law index is consistent with those of K98a and K98b within the error $(N=1.4 \pm 0.15$, Figure $1(\mathrm{c}))$.

The results stay the same even if a slightly higher resolution of $500 \mathrm{pc}$ is used $\left(N=1.3 \pm 0.06\right.$ and $A_{\mathrm{fit}}=-3.6 \pm 0.09$; Figure 2(a)). If the $\mathrm{BG}$ emission is subtracted from $\mathrm{H} \alpha$ and $24 \mu \mathrm{m}$ (Figure 2(b)), then the slope becomes even steeper $\left(N=1.8 \pm 0.10\right.$ and $\left.A_{\text {fit }}=-5.0 \pm 0.17\right)$, and more discrepant from the $\operatorname{CO}(J=2-1)$ results. This trend-a steeper slope after BG subtraction-is consistent with that found by Liu et al. (2011) with a smaller sample (two galaxies). This is caused by systematic variations of the BG emission; the fraction of the emission becomes larger at lower $\Sigma_{\mathrm{SFR}}$ regions. Some schemes of BG subtraction have been experimented with (e.g., Rahman et al. 2011; Liu et al. 2011; Leroy et al. 2012), but these systematics-greater reduction in flux in lower emission regions-are general. The BG subtraction makes the K-S law slope steeper as more BG emission is subtracted. (Note that a potential presence of diffuse $\mathrm{CO}$ emission was recently suggested (Pety et al. 2013), though the survival and excitation mechanism of $\mathrm{CO}$ in the diffuse environment is still a subject on debate. As an experiment, we also imposed a local BG subtraction in the $\mathrm{CO}$ images as well as in $\mathrm{H} \alpha$ and $24 \mu \mathrm{m}$ and found no significant change $(d N \sim 0.1)$.)

Our errors in $N$ and $A_{\text {fit }}$ are only statistical errors and do not include systematic errors. We adopted two fitting methods, FITEXY and OLS bisector, for comparisons with the previous work. Blanc et al. (2009) discussed the disadvantage of these methods and adopted a Monte Carlo fitting method for a more realistic treatment of systematics and accurate determination of the parameters and errors (see also Shetty et al. 2013). Such an analysis is beyond the scope of this Letter. We note, however, that in the end, our errors in $N(\sim 0.06$ and 0.10$)$ are of the same order as those from the sophisticated analysis $(\sim 0.05$; Blanc

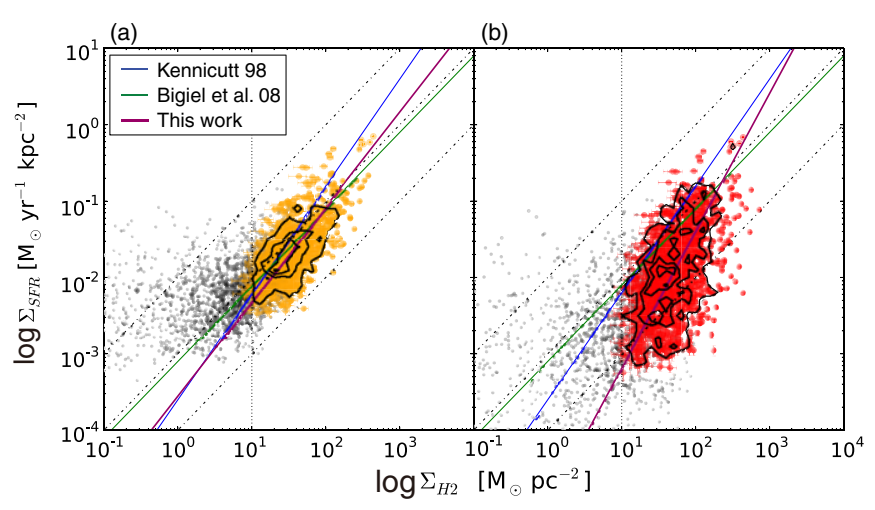

Figure 2. K-S plot on 500 pc scale, (a) without and (b) with BG subtraction, for our 10 sample galaxies. The dot-dashed lines are SFEs of $10^{-8}, 10^{-9}$, and $10^{-10} \mathrm{yr}^{-1}$, from top to bottom. The green and blue lines are the best fit of B08, and K98a and K98b, respectively. The light purple lines are the best-fit linear regressions through our data, yielding slopes of 1.3 (a) and 1.8 (b), respectively. The overlaid black contours are density distributions of the data points. We also plot all data points below $3 \sigma$ as black dots.

(A color version of this figure is available in the online journal.)

et al. 2009) while Leroy et al. (2013) reported a slightly larger error $\sim 1.5$.

All our results with the $\mathrm{CO}(J=1-0)$ data (at both $750 \mathrm{pc}$ and $500 \mathrm{pc}$ resolutions with and without BG subtractions) show the super-linear/power-law slope for the K-S law to be inconsistent with the previous finding using $\mathrm{CO}(J=2-1)$. $\mathrm{CO}(J=1-0)$ data provide the super-linear correlation of $N \sim 1.3-1.8$.

\section{DISCUSSION}

\subsection{The Cause of the Discrepancies}

We demonstrate that the $\operatorname{CO}(J=1-0)$ data provide a superlinear slope of the $\mathrm{K}-\mathrm{S}$ law, in contrast to the linear slope obtained by the $\operatorname{CO}(J=2-1)$ studies, even when the diffuse $\mathrm{BG}$ emission is not subtracted from SFR tracer images. We here discuss possible causes of the discrepancy: differences in SFR tracers (i.e., $\mathrm{H} \alpha+24 \mu \mathrm{m}$ versus FUV+24 $\mu \mathrm{m}$ ), and differences in molecular gas density tracers (i.e., $\mathrm{CO} J=1-0$ versus $2-1$ ).

B08 estimated SFR using FUV and $24 \mu \mathrm{m}$, instead of $\mathrm{H} \alpha$ and $24 \mu \mathrm{m}$. The two SFRs are compared and found consistent by previous studies. For example, some studies (B08; Leroy et al. 2008, 2012; Liu et al. 2011) demonstrated that the $\Sigma_{\text {SFR }}$ 
from $\mathrm{FUV}+24 \mu \mathrm{m}$ are equal to those derived from $\mathrm{H} \alpha+24 \mu \mathrm{m}$ by Calzetti et al. (2007), though they did not account for the BG emission. They also noted that the correlation becomes poorer in the range of very low $\operatorname{SFR}\left(\Sigma_{\mathrm{SFR}} \leqslant 10^{-3} M_{\odot} \mathrm{yr}^{-1} \mathrm{kpc}^{-2}\right)$. Our analysis does not suffer from this, since all the points in Figure 2 are above this boundary SFR. These comparisons in the previous studies give us confidence that the differences in SFR tracers are unlikely to be the cause of the discrepancy between our results and those of B08.

The difference between $\operatorname{CO}(J=1-0)$ and $\operatorname{CO}(J=2-1)$ is likely a contributor to the discrepancy both empirically and due to physical considerations. The observed kinetic temperature of molecular gas is typically $\sim 10 \mathrm{~K}$ (Scoville \& Sanders 1987), which is above the level energy temperature of $5.5 \mathrm{~K}$ for the $J=1$, but below the temperature of $16.5 \mathrm{~K}$ for $J=2$. Therefore, a slight change in gas kinetic temperature affects the excitation for $\mathrm{CO}(J=2-1)$ emission significantly. Furthermore, the change of the molecular gas volume density also affects the excitation for $\operatorname{CO}(J=2-1)$. The different critical densities of the transitions $\left(\sim 10^{3-4} \mathrm{~cm}^{-3}\right.$ and a few $\times 10^{2} \mathrm{~cm}^{-3}$ for $J=$ $2-1$ and $1-0$, respectively) make their ratio sensitive to local gas density. In fact, the ratio of $\mathrm{CO}(J=2-1)$ to $\mathrm{CO}(J=1-0)$ varies systematically with SFR and $\Sigma_{\text {gas }}$ in M 51 (Koda et al. 2012; Vlahakis et al. 2013) and in the Galaxy (e.g., Sakamoto et al. 1995, 1997; Sawada et al. 2001), although this systematic trend was often buried in noise (Leroy et al. 2013). The higher SFR and $\Sigma_{\text {gas }}$ are, the greater the ratio is; this systematic trend directly affects the study of the K-S law using $\mathrm{CO}(2-1)$.

Previous studies suggested a change of the K-S law index when a higher $\mathrm{CO}$ rotational transition is used (e.g., Narayanan et al. 2008; Bayet et al. 2009). Indeed, the excitation condition depends on the volume density and temperature; for example, the fraction of the gas above critical density of a line excitation is one of the key determinants for the slope of the K-S law (Narayanan et al. 2008). If the critical density for a line emission is high and close to the conditions of star-forming gas, then that line emission can naturally show a linear relationship with SFR. Therefore, a higher- $J$ CO line transition tends to show a linear slope ( $N=1$, e.g., Iono et al. 2009). On the other hand, $\operatorname{CO}(J=$ $1-0)$ has a relatively lower critical density for excitation, tracing the bulk molecular gas. Our results for super-linear correlation by $\mathrm{CO}(J=1-0)$ imply that the star formation efficiency (SFE) depends on the average gas surface density over $\sim 1 \mathrm{kpc}$ scale, indicating the importance of environments around GMC on star formation.

\subsection{Implications for Star Formation}

The super-linear slope of the K-S law perhaps indicates that the process of star formation is nonlinear when it is seen on $\geqslant 500$ pc scales. Our results with $\mathrm{CO}(J=1-0)$ suggest a superlinear correlation both at $500 \mathrm{pc}$ and $750 \mathrm{pc}$ scales whether or not the diffuse BG emission is subtracted from SFR tracer images. These spatial scales are much larger than the typical GMC size ( $\sim 40$ pc; Scoville \& Sanders 1987). The super-linear correlation at these scales may suggest some nonlinearity in processes of dense gas core formation and star formation in individual GMCs (e.g., Calzetti et al. 2012).

A relatively simple model of star formation can reproduce the super-linear slope $N$. The self-gravity of the gas naturally explains $N=1.5$ since the free-fall time $\tau_{\mathrm{ff}}$ is proportional to the inverse of the square root of the density. $\Sigma_{\mathrm{SFR}}$ should be proportional to $\Sigma_{\text {gas }} / \tau_{\text {ff }}$ (e.g., Krumholz \& McKee 2005; Krumholz et al. 2012). Gravitational instability can play a role at larger scales. For example, a consideration of Toomre's $Q$ instability parameter in rotating galactic disks also leads to $N=$ 1.5 (e.g., Silk 1997; Elmegreen 2002). A steeper slope $N=2$ is possible as well if star formation is triggered by cloud-cloud collisions (e.g., Tan 2000, 2010). Our result suggests that the SFE is not constant across galactic disks, though the non-linear process that plays a dominant role in driving star formation remains difficult to identify through our works.

We are grateful to the referee for constructive comments to improve this Letter. We thank to Frank Bigiel, Rahul Shetty, and Junichi Baba for discussions, Yasutaka Kurono for helping us to combine our $\mathrm{CO}(J=1-0)$ data, and James Barrett for helpful comments on the English. We also thank the SINGS team, the NRO staff for NRO45 observations, and the CARMA staff for CARMA observations. Support for CARMA construction was derived from the Gordon and Betty Moore Foundation, the Kenneth T. and Eileen L. Norris Foundation, the James S. McDonnell Foundation, the Associates of the California Institute of Technology, the University of Chicago, the states of California, Illinois, and Maryland, and the National Science Foundation. Ongoing CARMA development and operations are supported by the National Science Foundation under a cooperative agreement and by the CARMA partner universities. This research was partially supported by Hayakawa Yukio Foundation. J.K. acknowledges support from the NSF through grant AST-1211680.

\section{REFERENCES}

Bayet, E., Gerin, M., Phillips, T. G., \& Contursi, A. 2009, MNRAS, 399, 264 Bigiel, F., Leroy, A., Walter, F., et al. 2008, AJ, 136, 2846 (B08)

Blanc, G. A., Heiderman, A., Gebhardt, K., Evans, N. J., II, \& Adams, J. 2009, ApJ, 704, 842

Bolatto, A. D., Leroy, A. K., Rosolowsky, E., Walter, F., \& Blitz, L. 2008, ApJ, 686,948

Bolatto, A. D., Wolfire, M., \& Leroy, A. K. 2013, arXiv:1301.3498

Calzetti, D., Kennicutt, R. C., Engelbracht, C. W., et al. 2007, ApJ, 666, 870

Calzetti, D., Liu, G., \& Koda, J. 2012, ApJ, 752, 98

Dale, D. A., Cohen, S. A., Johnson, L. C., et al. 2009, ApJ, 703, 517

Dame, T. M., Hartmann, D., \& Thaddeus, P. 2001, ApJ, 547, 792

Donovan Meyer, J., Koda, J., Momose, R., et al. 2012, ApJ, 744, 42

Donovan Meyer, J., Koda, J., Momose, R., et al. 2013, ApJ, accepted (arXiv:1305.5275)

Draine, B. T., Dale, D. A., Bendo, G., et al. 2007, ApJ, 663, 866

Draine, B. T., \& Li, A. 2007, ApJ, 657, 810

Elmegreen, B. G. 2002, ApJ, 577, 206

Ferguson, A. M. N., Wyse, R. F. G., Gallagher, J. S., III., \& Hunter, D. A. 1996, AJ, 111, 2265

Iono, D., Wilson, C. D., Yun, M. S., et al. 2009, ApJ, 695, 1537

Isobe, T., Feigelson, E. D., Akritas, M. G., \& Babu, G. J. 1990, ApJ, 364, 104

Kennicutt, R. C., Jr. 1998a, ARA\&A, 36, 189,(K98a)

Kennicutt, R. C., Jr. 1998b, ApJ, 498, 541, (K98b)

Kennicutt, R. C., Jr., Armus, L., Bendo, G., et al. 2003, PASP, 115, 928

Kennicutt, R. C., Jr., \& Evans, N. J., II 2012, ARA\&A, 50, 531

Kennicutt, R. C., Jr., Calzetti, D., Walter, F., et al. 2007, ApJ, 671, 333

Knapen, J. H., Stedman, S., Bramich, D. M., Folkes, S. L., \& Bradley, T. R. 2004, A\&A, 426, 1135

Koda, J., Sawada, T., Wright, M. C. H., et al. 2011, ApJS, 193, 19

Koda, J., Scoville, N., Hasegawa, T., et al. 2012, ApJ, 761, 41

Krumholz, M. R., Dekel, A., \& McKee, C. F. 2012, ApJ, 745, 69

Krumholz, M. R., \& McKee, C. F. 2005, ApJ, 630, 250

Leroy, A. K., Bigiel, F., de Blok, W. J. G., et al. 2012, AJ, 144, 3

Leroy, A. K., Walter, F., Brinks, E., et al. 2008, AJ, 136, 2782

Leroy, A. K., Walter, F., Sandstrom, K., et al. 2013, AJ, 146, 19

Liu, G., Koda, J., Calzetti, D., Fukuhara, M., \& Momose, R. 2011, ApJ, 735, 63 Moustakas, J., Kennicutt, R. C., Jr., Tremonti, C. A., et al. 2010, ApJS, 190, 233

Narayanan, D., Cox, T. J., Shirley, Y., et al. 2008, ApJ, 684, 996

Pety, J., Schinnerer, E., Leroy, A. K., et al. 2013, arXiv:1304.1396 
Press, W. H., Teukolsky, S. A., Vetterling, W. T., \& Flannery, B. P. 1992, Numerical Recipes in FORTRAN. The Art of Scientific Computing (Cambridge: Cambridge Univ. Press)

Rahman, N., Bolatto, A. D., Xue, R., et al. 2012, ApJ, 745, 183

Rahman, N., Bolatto, A. D., Wong, T., et al. 2011, ApJ, 730, 72

Sakamoto, S., Hasegawa, T., Handa, T., Hayashi, M., \& Oka, T. 1997, ApJ, 486,276

Sakamoto, S., Hasegawa, T., Hayashi, M., Handa, T., \& Oka, T. 1995, ApJS, 100,125

Sawada, T., Hasegawa, T., Handa, T., et al. 2001, ApJS, 136, 189

Schmidt, M. 1959, ApJ, 129, 243

Schruba, A., Leroy, A. K., Walter, F., et al. 2011, AJ, 142, 37
Scoville, N. Z., Polletta, M., Ewald, S., et al. 2001, AJ, 122, 3017

Scoville, N. Z., \& Sanders, D. B. 1987, ASSL, 134, 21

Silk, J. 1997, ApJ, 481, 703

Shetty, B., Kelly, B. C., \& Bigiel, F. 2013, MNRAS, 430, 288

Strong, A. W., \& Mattox, J. R. 1996, A\&A, 308, L21

Tan, J. C. 2000, ApJ, 536, 173

Tan, J. C. 2010, ApJL, 710, L88

Thilker, D. A., Braun, R., \& Walterbos, R. A. M. 2000, AJ, 120, 3070

Verley, S., Corbelli, E., Giovanardi, C., \& Hunt, L. K. 2009, A\&A, 493, 453

Vlahakis, C., van der Werf, P., Israel, F. P., \& Tilanus, R. P. J. 2013, MNRAS, in press (arXiv:1304.7408)

Wong, T., \& Blitz, L. 2002, ApJ, 569, 157 